\title{
Natural boundaries for Euler products of Igusa zeta functions of elliptic curves.
}

\author{
Marcus du Sautoy*
}

May 24, 2018

\begin{abstract}
We study the analytic behaviour of adelic versions of Igusa integrals given by integer polynomials defining elliptic curves.
\end{abstract}

2010 Mathematics Subject Classification: 11M41

\section{Contents}

1 Introduction

2 Some polynomial identities

3 Some meromorphic functions 6

3.1 L-functions of Hecke-grossencharacters . . . . . . . . . . . . 6

3.2 Symmetric power L-functions . . . . . . . . . . . . 7

4 Meromorphic continuation 8

5 Natural boundaries 12

\section{Introduction}

Let $f(x, y) \in \mathbb{Z}[x, y]$ be a nonsingular integer polynomial defining an elliptic curve $E$. The Igusa zeta function of $f$ at the prime $p$ is defined as:

$$
I_{E}(s, p)=\int_{\mathbb{Z}_{p}^{2}}|f(x, y)|_{p}^{s} d \mu
$$

where ||$_{p}$ stands for the p-adic absolute value and $d \mu$ for the Haar-measure on $\mathbb{Q}_{p}^{2}$ normalized such that $\mathbb{Z}_{p}^{2} \subset \mathbb{Q}_{p}^{2}$ has measure equal to 1 .

${ }^{*}$ Mathematical Institute, Andrew Wiles Building, Woodstock Road, Oxford OX2 6GG, UK. dusautoy@maths.ox.ac.uk 
Let $S(E)$ be the set of those primes $p$ for which the reduction of $f(x, y)$ modulo $p$ becomes singular. Note that $S(E)$ is a finite set of primes. For the primes $p \notin S(E)$ the integral (11) can be computed as:

$$
I_{E}(s, p)=\left(1-p^{-2} C_{p}\right)\left(1+\lambda_{p} \frac{p^{-(s+1)}}{\left(1-p^{-(s+1)}\right)}\right)
$$

(see Proposition 5) where the numbers $C_{p}$ and $\lambda_{p}$ are given by:

$$
\begin{gathered}
C_{p}=\operatorname{card}\{(x, y) \in \mathbb{Z} / p \mathbb{Z} \quad \mid f(x, y)=0 \bmod p\}, \\
\lambda_{p}=\frac{(p-1) C_{p}}{\left(p^{2}-C_{p}\right)} .
\end{gathered}
$$

Following Ono 12 we form the Euler-product

$$
I_{E}(s)=\prod_{p \notin S(E)} I_{E}(s, p)\left(1-p^{-2} C_{p}\right)^{-1}
$$

which have their constant coefficients equal to 1 .

We call $I_{E}(s)$ the global Igusa-zeta function of the elliptic curve given by $f$. Given this notation we show:

Theorem 1 (i) The Euler product $I_{E}(s)$ converges for $s \in \mathbb{C}$ with $\Re(s)>0$,

(ii) $I_{E}(s)$ has a meromorphic continuation to the region $\Re(s)>-3 / 2$,

(iii) (assuming GRH) the line $\Re(s)=-3 / 2$ is a natural boundary for $I_{E}(s)$ beyond which no further meromorphic continuation is possible.

In the case that $E$ has no complex multiplication, the result depends on the recent proof of the Sato-Tate conjectures together with the associated proof about the meromorphic continuation of symmetric power $L$-functions (see [1, 9] and 14).

Interest in proving such a theorem arose from considerations of meromorphic continuation and natural boundaries for zeta functions of groups and rings. 22.

Grunewald, Segal and Smith introduced the notion of the zeta function of a group $G$ in 8 :

$$
\zeta_{\bar{G}}^{\leq}(s)=\sum_{H \leq G}|G: H|^{-s}=\sum_{n=1}^{\infty} a_{n}^{\leq}(G) n^{-s}
$$

where $a_{\bar{n}} \leq(G)$ denotes the number of subgroups of index $n$ in $G$.

They proved that for finitely generated, torsion-free nilpotent groups the global zeta function can be written as an Euler product of local factors which are rational functions in $p^{-s}$ :

$$
\begin{aligned}
\zeta_{\bar{G}}^{\leq}(s) & =\prod_{p \text { prime }} \zeta_{\bar{G}, p}^{\leq}(s) \\
& =\prod_{p \text { prime }} Z_{p}^{\leq}\left(p, p^{-s}\right)
\end{aligned}
$$

where for each prime $p, \zeta_{\bar{G}, p}^{\leq}(s)=\sum_{n=0}^{\infty} a_{p^{n}}^{\leq}(G) p^{-n s}$ and $Z_{\bar{p}}^{\leq}(X, Y) \in \mathbb{Q}(X, Y)$. 
Similar definitions and results were also obtained for the zeta function $\zeta_{G}^{\triangleleft}(s)$ counting normal subgroups. Analogous results also hold for zeta functions counting ideals or subalgebras in finite dimensional Lie algebras.

In [3] the author and Grunewald were able to prove that these zeta functions have some meromorphic continuation beyond their radius of convergence.

Theorem 2 Let $G$ be a finitely generated nilpotent group. Then the abscissa of convergence $\alpha(G)$ of $\zeta_{G}(s)$ is a rational number and $\zeta_{G}(s)$ has a meromorphic continuation to the right half-plane $\operatorname{Re}(s)>\alpha(G)-\delta$ for suitable $\delta>0$.

The challenge was then to determine how far one could meromorphically continue such functions or whether at some point you encountered a natural boundary beyond which no continuation was possible. Many of the early examples of calculations of these functions revealed a uniform behaviour of the rational functions as one varied the prime $p$. In particular the zeta function of a nilpotent group $G$ is called uniform if there exists $W(X, Y) \in \mathbb{Q}(X, Y)$ such that for almost all primes $p$

$$
\zeta_{\bar{G}, p}^{\leq}(s)=W\left(p, p^{-s}\right) .
$$

In Chapter 5 of [2] given a two variable polynomial $W(X, Y)$, we provided a criterion for the Euler product $\prod_{p \text { prime }} W\left(p, p^{-s}\right)$ to have a natural boundary beyond which one cannot continue the function meromorphically. This partially answered a conjecture characterizing those polynomials which do admit meromorphic continuation to the whole complex plane, generalizing a result of Estermann's for one variable polynomials [6.

However in 4 and 5] we proved that not all finitely generated nilpotent groups have uniform zeta functions. In particular we proved the following:

Theorem 3 Let $L$ be the class two nilpotent Lie algebra over $\mathbb{Z}$ of dimension 9 as a free $\mathbb{Z}$-module given by the following presentation:

$L=\left\langle\begin{array}{c}x_{1}, x_{2}, x_{3}, x_{4}, x_{5}, x_{6}, y_{1}, y_{2}, y_{3}:\left(x_{1}, x_{4}\right)=y_{3},\left(x_{1}, x_{5}\right)=y_{1},\left(x_{1}, x_{6}\right)=y_{2} \\ \left(x_{2}, x_{4}\right)=y_{1},\left(x_{2}, x_{5}\right)=y_{3},\left(x_{3}, x_{4}\right)=y_{2},\left(x_{3}, x_{6}\right)=y_{1}\end{array}\right\rangle$

where all other commutators are defined to be 0 . Let $E$ be the elliptic curve $Y^{2}=X^{3}-X$. Then there exist two non zero rational functions $P_{1}(X, Y)$ and $P_{2}(X, Y) \in \mathbb{Q}(X, Y)$ such that for almost all primes $p$ :

$$
\zeta_{L_{p}}^{\triangleleft}(s)=P_{1}\left(p, p^{-s}\right)+\left|E\left(\mathbb{F}_{p}\right)\right| P_{2}\left(p, p^{-s}\right) .
$$

Interest therefore turned to whether the global zeta function of this example whose local factors depend on the number of points on an elliptic curve might have natural boundaries beyond which no meromorphic continuation is possible.

Given that the main tool in proving these results is the use of $p$-adic integrals similar to those defining the Igusa zeta function it seemed apposite to first consider the global nature of a function defined as an Euler product of local Igusa zeta functions. Such global functions have received little attention beyond an initial paper by Ono 12 where some meromorphic continuation of this global zeta function is demonstrated. This paper can be considered as a continuation of that early paper of Ono's, establishing that in general these global Igusa functions have natural boundaries.

I should like to dedicate this paper to the memory of Fritz Grunewald with whom I discussed many of the ideas at the heart of this paper and beyond. 


\section{Some polynomial identities}

This section contains several polynomial identities which will be important for the meromorphic continuation of the global Igusa zeta functions.

We write $\mathbb{N}$ for the natural numbers and $\mathbb{N}_{0}$ with zero included. Let $\mathcal{L}$ be the set of 3-tuples $(r, n, m) \in \mathbb{N}_{0}^{2} \times \mathbb{N}$ with the property that $(r+2 n) / 2 m=1 / 2$ or equivalently with $r+2 n=m$. For each $M \in \mathbb{N}$ let $\mathcal{L}_{M}$ denote the finite set of $(r, n, m) \in \mathcal{L}$ for which $m<M$. For $(r, n, m) \in \mathcal{L}$ we define its weight to be:

$$
\Theta(r, n, m):=\frac{r+2 n+2}{2 m}=\frac{1}{2}+\frac{1}{m} .
$$

We consider here the free commutative $\mathbb{Z}$-algebra $R$ generated by $u, v, X, Y$ with the single relation $u v=X$, that is

$$
R:=\mathbb{Z}\langle u, v, X, Y \quad \mid u v=X\rangle .
$$

Every monomial in $u, v, X, Y$ in $R$ is equal to $u^{r_{1}} v^{r_{2}} X^{n} Y^{m}$ with $r_{1}, r_{2}, n, m \in$ $\mathbb{N}_{0}$ and with at least one of the $r_{1}, r_{2}$ equal to zero. Every $Q \in R$ which is symmetric in $u, v$ can be written as

$$
Q=\sum_{(r, n, m) \in \mathbb{N}_{0}^{3}} a_{Q,(r, n, m)}\left(u^{r}+v^{r}\right) X^{n} Y^{m}
$$

with uniquely determined coefficients $a_{Q,(r, n, m)} \in \mathbb{Z}, a_{Q,(r, n, m)}=0$ for almost all $(r, n, m) \in \mathbb{N}_{0}^{3}$.

For $(r, n, m) \in \mathcal{L}$ and $\varepsilon \in\{ \pm 1\}$ with $r \neq 0$ we define:

$$
P_{(r, n, m)}^{[\varepsilon]}:=1-\varepsilon\left(u^{r}+v^{r}\right) X^{n} Y^{m}+X^{r+2 n} Y^{2 m}
$$

For $(r, n, m) \in \mathcal{L}$ with $r=0$ and $\varepsilon \in\{ \pm 1\}$ we define:

$$
P_{(0, n, m)}^{[\varepsilon]}:=1-\varepsilon X^{n} Y^{m}
$$

We establish now the following cyclotomic expansion of $1-u Y-v Y$.

Lemma 1 For $w=(r, n, m) \in \mathcal{L}$ there exist integers $c(w) \in \mathbb{N}_{0}$ and $\varepsilon(w) \in$ $\{-1,1\}$ with the property that for $M \in \mathbb{N}$

$$
1-u Y-v Y=\left(\prod_{w \in \mathcal{L}_{M}}\left(P_{w}^{[\varepsilon(w)]}\right)^{c(w)}\right)+W_{M}(u, v, X, Y)
$$

where $W_{M}$ is symmetric with respect to $u, v$ and $(r, n, m) \in \mathcal{L} \backslash \mathcal{L}_{M}$ for every $(r, n, m) \in \mathbb{N}_{0}^{3}$ for which the coefficient $a_{W_{M},(r, n, m)}$ from (8) is non-zero.

Before we give the proof of the lemma we shall give several examples

$$
\begin{gathered}
\mathrm{M}=2 \\
1-u Y-v Y=P_{(1,0,1)}^{[1]}-X Y^{2}, \\
\mathrm{M}=3 \\
1-u Y-v Y=P_{(1,0,1)}^{[1]} P_{(0,1,2)}^{[1]}-(u+v) X Y^{3}+X^{2} Y^{4},
\end{gathered}
$$




$$
\begin{gathered}
\mathrm{M}=4 \\
1-u Y-v Y=P_{(1,0,1)}^{[1]} P_{(0,1,2)}^{[1]} P_{(1,1,3)}^{[1]}+\left(u^{2}+v^{2}\right) X^{2} Y^{6} \\
-\left(u^{2}+v^{2}\right) X Y^{4}-(u+v) X^{4} Y^{9}+X^{5} Y^{10}+X^{3} Y^{6}-X^{2} Y^{4} .
\end{gathered}
$$

We note the following identity: for $n \geq m$

$$
\left(u^{n}+v^{n}\right)\left(u^{m}+v^{m}\right)=\left(u^{n+m}+v^{n+m}\right)+\left(u^{n-m}+v^{n-m}\right) X^{m} .
$$

Proof of Lemma 1 This follows by induction on $M$. We start the induction by writing:

$$
1-u Y-v Y=P_{(1,0,1)}^{[1]}-X Y^{2}
$$

To deal with the inductive step we need to show how to remove a term of the form $a_{r, n, M}\left(u^{r}+v^{r}\right) X^{n} Y^{m}$ in $W_{M}(u, v, X, Y)$ at the expense of introducing things of the form $a_{r, n, m}\left(u^{r}+v^{r}\right) X^{n} Y^{m}$ with $m>M$. If $r>0$, then we introduce a new term

$$
\left(1+(-1)^{\varepsilon_{r, n, M}}\left(u^{r}+v^{r}\right) X^{n} Y^{M}+X^{r+2 n} Y^{2 M}\right)^{c_{r, n, M}}
$$

where $a_{r, n, M}=(-1)^{\varepsilon_{r, n, M}} c_{r, n, M}$ and $c_{r, n, M}>0$. The error terms that we must introduce into $W_{M+1}(u, v, X, Y)$ are then of the form

$$
c\left(u^{r_{1}}+v^{r_{1}}\right) \cdots\left(u^{r_{l}}+v^{r_{l}}\right) X^{i} Y^{m}
$$

where $m>M$. We can rewrite this using the identity (11) as a sum of terms of the form $c^{\prime}\left(u^{r}+v^{r}\right) X^{n} Y^{m}$ with the property that $(r+2 n) / 2 m=1 / 2$. (Note that if $a / b=c / d=1 / 2$ then $(a+c) /(b+d)=1 / 2$. $)$

Using Lemma 1 we define for $M \in \mathbb{N}$

$$
Q_{M}(u, v, X, Y):=\prod_{w \in \mathcal{L}_{M}}\left(P_{w}^{[\varepsilon(w)]}\right)^{c(w)} \in R
$$

Suppose $S$ is a finite set of prime numbers and for every prime $p \notin S$ we are given a $\pi_{p} \in \mathbb{C}$ with $\pi_{p} \overline{\pi_{p}}=p$. We consider then the Euler products

$$
\begin{gathered}
Z_{M}^{1}(s):=\prod_{p \notin S}\left(1+W_{M}\left(\pi_{p}, \overline{\pi_{p}}, p, p^{-s}\right)\right. \\
Z_{M}^{2}(s):=\prod_{p \notin S}\left(1+\frac{W_{M}\left(\pi_{p}, \overline{\pi_{p}}, p, p^{-s}\right)}{Q_{M}\left(\pi_{p}, \overline{\pi_{p}}, p, p^{-s}\right)}\right)
\end{gathered}
$$

Lemma 2 Both the Euler products $Z_{M}^{1}(s)$ and $Z_{M}^{2}(s)$ converge absolutely for $s \in \mathbb{C}$ with $\Re(s)>1 / 2+1 / M$

For $n \in \mathbb{N}$ we define:

$$
S^{[n]}:=\prod_{i=0}^{n}\left(1-u^{i} v^{n-i} Y\right)
$$


We have the recursion relations:

$$
S^{[2 n]}=P_{(0, n, 1)}^{[1]} \cdot \prod_{i=1}^{n} P_{2 i, n-i, 1}^{[1]}
$$

We have the recursion relations:

$$
S^{[2 n-1]}=\prod_{i=1}^{n} P_{2 i-1, n-i, 1}^{[1]}
$$

\section{Some meromorphic functions}

This section accumulates certain facts about the analytic properties of of Lfunctions of Hecke-Groessencharacters and for symmetric power L-functions

\subsection{L-functions of Hecke-grossencharacters}

Our basic reference for the terminology and theory of quasi-characters of Ideleclass groups is 10 .

Let $K \subset \mathbb{C}$ be an imaginary quadratic number field, $\mathbb{A}_{K}^{*}$ its group of ideles and $J_{K}:=K^{*} \backslash \mathbb{A}_{k}^{*}$ its group of idele classes. We identify the set of finite places of $K$ with the set of prime ideals in the ring of integers $\mathcal{O}$ of $K$. Given such a prime ideal $\wp$ we choose a generator $\pi_{\wp}$ of the maximal ideal of the completion $\mathcal{O}_{\wp}$. We define $[\wp] \in J_{K}$ to be the class of the idele which is 1 at all places except for $\wp$ where it is $\pi_{\wp}$.

Let

$$
\chi: J_{K} \rightarrow \mathbb{C}^{*}
$$

be a (continuous) quasi-character and $S(\chi)$ the set of non-archimedian places where $\chi$ is ramified. The Hecke L-series of $\chi$ is defined as

$$
L(\chi, s):=\prod_{\wp \notin S(\chi)}\left(1-\chi([\wp]) N(\wp)^{-s}\right)^{-1}
$$

Proposition 3 Let $\chi$ be a quasi-character of the idele-class group with the property that $|\chi([\wp])|=N(\wp)^{\frac{1}{2}}$ for all $\wp \notin S(\chi)$. Assume also that $r$ is a natural number. The following hold:

(i) The L-function $L\left(\chi^{r}, s\right)$ converges for $\Re(s)>2 r$.

(ii) The L-function $L\left(\chi^{r}, s\right)$ extends to a holomorphic function on all of $\mathbb{C}$.

(iii) The zeroes of the extended L-function $L\left(\chi^{r}, s\right)$ in $\{s \in \mathbb{C} \mid \Re(s)<0\}$ lie on the real axis.

These functions will be sufficient to do meromorphic continuation of our global Igusa zeta function in the case that the elliptic curve has complex multiplication. Otherwise we need the following section. 


\subsection{Symmetric power L-functions}

We report here on the recent progress proving results about the meromorphic properties of the symmetric power L-functions (see [1, [9] and [14]). We also refer to [13] for another good reference.

We write $\mathbb{A}$ for the ring of adeles of $\mathbb{Q}$. Let further $S$ be a finite set of places including the archimedian place. We shall identify the non-archimedian places with the corresponding prime numbers. Let $\omega$ be a cuspidal representation of $\mathbf{G L}(2, \mathbb{A})$. Assume that the associated local representation $\omega_{p}$ is of class 1 and also tempered for all $p \notin S$. For $p \notin S$ let

$$
t_{p}=\left(\begin{array}{cc}
\alpha_{p} & 0 \\
0 & \beta_{p}
\end{array}\right) \in \mathbf{G L}(2, \mathbb{C})
$$

be the conjugacy class parametrizing $\omega_{p}$. We use here the normalisation chosen in [13, Section 5, that is $\left|\alpha_{p}\right|=\left|\beta_{p}\right|=1$ and $\alpha_{p} \beta_{p}=1$.

Let $m$ be a natural number. For $p \notin S$ the local factor of the $m$-th symmetric power L-function attached to $\omega$ is defined by:

$$
L\left(s, \omega_{p}, m\right):=\prod_{i=0}^{m}\left(1-\alpha_{p}^{i} \beta_{p}^{m-i} p^{-s}\right)^{-1}
$$

The (partial) global $m$-th symmetric power L-function is defined as the Euler product:

$$
L(s, \omega, m):=\prod_{p \notin S} L\left(s, \omega_{p}, m\right)
$$

The Dirichlet series $L(s, \omega, m)$ converges for $\Re(s)>1$. The results of [1], 9 ] and [14] prove the following:

Theorem $4 L(s, \omega, m)$ has a meromorphic continuation to all of $\mathbb{C}$ for every $m \in \mathbb{N}$.

Assume now that the automorphic represention $\omega$ is attached to a holomorphic cusp form $g$ of weight $k$ for the congruence subgroup

$$
\begin{gathered}
\Gamma_{0}(N):=\left\{\left(\begin{array}{ll}
a & b \\
c & d
\end{array}\right) \in \mathbf{S L}(2, \mathbb{Z}) \mid c \equiv 0 \bmod N\right\} \\
\alpha_{p}=\frac{\pi_{p}}{p^{k-1 / 2}}, \quad \alpha_{p}=\frac{\bar{\pi}_{p}}{p^{k-1 / 2}}
\end{gathered}
$$

Proposition 4 Let $g$ be a holomorphic cusp form of weight 2 for $\Gamma_{0}(N)$. Define for $r \in \mathbb{N}$ and $\varepsilon \in \pm 1$ :

$$
Z_{r}^{[\varepsilon]}(s)=\prod_{p \notin S(g)} Z_{r, p}^{[\varepsilon]}(s)
$$

where

$$
Z_{r, p}^{[\varepsilon]}(s)=1-\varepsilon\left(\pi_{p}^{r}+\bar{\pi}_{p}^{r}\right) p^{-s}+p^{r-2 s} .
$$

Then $Z_{r}^{[\varepsilon]}(s)$ is a meromorphic function on $\mathbb{C}$.

For $\varepsilon=1$ follows from the recursive relations of the previous section.

For $\varepsilon=-1$ use the fact that

$$
Z_{r, p}^{[-1]}(s)=Z_{2 r, p}^{[1]}(2 s) / Z_{r, p}^{[1]}(s)
$$




\section{Meromorphic continuation}

Let $f(x, y) \in \mathbb{Z}[x, y]$ denote a non-singular polynomial and let $S(f)$ be the set of primes for which the reduction of $f$ in $\mathbb{Z} / p \mathbb{Z}$ becomes singular. As in the introduction we define the local Igusa integral at the prime $p$ by:

$$
I_{f}(s, p)=\int_{\mathbb{Z}_{p}^{2}}|f(x, y)|^{s}|d x||d y| .
$$

We have:

Proposition 5 Let $f(x, y) \in \mathbb{Z}[x, y]$ be a non-singular polynomial and $p \notin S(f)$ then

$$
I_{f}(s, p)=\left(1-p^{-2} C_{p}\right)\left(1+\lambda_{p} \frac{p^{-(s+1)}}{\left(1-p^{-(s+1)}\right)}\right)
$$

where

$$
\lambda_{p}=\frac{(p-1) C_{p}}{\left(p^{2}-C_{p}\right)} .
$$

and $C_{p}=\operatorname{card}\{(x, y) \in \mathbb{Z} / p \mathbb{Z} \quad \mid f(x, y)=0 \bmod p\}$.

Proof. Let

$$
\begin{array}{c|c}
E_{0}=\left\{(x, y) \in \mathbb{Z}_{p}^{2}\right. & \mid f(x, y) \neq 0 \bmod p\} \\
E_{1}=\left\{(x, y) \in \mathbb{Z}_{p}^{2}\right. & \mid f(x, y)=0 \bmod p\} \\
E_{n}=\left\{(x, y) \in \mathbb{Z}_{p}^{2}\right. & \left.f(x, y)=0 \bmod p^{n}\right\}
\end{array}
$$

Let

$$
\tilde{E}_{n}=\left\{(x, y) \in\left(\mathbb{Z}_{p} / p^{n} \mathbb{Z}_{p}\right)^{2} \mid f(x, y)=0 \bmod p^{n}\right\}
$$

and put $C_{p^{n}}=\operatorname{card} \tilde{E_{n}}$. Since $f(x, y)$ is non-singular $C_{p^{n}}=p^{n-1} C_{p}$.

$$
\begin{aligned}
I & =\sum_{n=0}^{\infty} p^{-n s} \cdot \int_{E_{n} \backslash E_{n+1}} \\
& =p^{-2} C_{1}+\sum_{n=1}^{\infty} p^{-n s} \cdot\left(C_{p^{n}} p^{-2 n}-C_{p^{n+1}} p^{-2 n-2}\right) \\
& =p^{-2} C_{1}+\sum_{n=1}^{\infty} p^{-n s} \cdot C_{p} p^{(n-1)-2 n}\left(1-p^{-1}\right) \\
& =\left(1-p^{-2} C_{p}\right)+p^{-1}\left(1-p^{-1}\right) \frac{p^{-(s+1)}}{\left(1-p^{-(s+1)}\right.} \\
& =\left(1-p^{-2} C_{p}\right)\left(1+\lambda_{p} \frac{p^{-(s+1)}}{\left(1-p^{-(s+1)}\right)}\right)
\end{aligned}
$$

where

$$
\lambda_{p}=\frac{(p-1) C_{p}}{\left(p^{2}-C_{p}\right)}
$$


The global Igusa zeta function of $f$ is then

$$
I_{f}(s)=\prod_{p \notin S(f)} I_{f}(s, p)\left(1-p^{-2} C_{p}\right)^{-1}
$$

where the constant coefficient of the local factor $I_{f}(s, p)$ has been normalized to be 1 . Now let's set

$$
C_{p}=p-a_{p}
$$

It is well known that

$$
\left|a_{p}\right| \leq 2 g(f) p^{1 / 2} .
$$

for every $p \notin S(f)$ with $g(f)$ being the genus of the complex curve defined by $f$.

Proposition 6 Let $f(x, y) \in \mathbb{Z}[x, y]$ be a non-singular polynomial then the abscissa of convergence of $I_{f}(s)$ is 0 .

Proof. Recall that

$$
\prod_{p}\left(1+r_{p}\right)
$$

is absolutely convergent if and only $\sum_{p}\left|r_{p}\right|$ converges. Also $\sum_{p}\left|p^{-s}\right|$ converges if and only if $\Re(s)>1$.

We have

$$
1+\lambda_{p} \frac{p^{-(s+1)}}{\left(1-p^{-(s+1)}\right)}=1+\frac{p^{-(s+1)}}{\left(1-p^{-(s+1)}\right)}-\frac{p a_{p}}{\left(p^{2}-p+a_{p}\right)} \frac{p^{-(s+1)}}{\left(1-p^{-(s+1)}\right)} .
$$

Using the fact that $\left|a_{p}\right| \leq 2 p^{1 / 2}$ we deduce that the abscissa of convergence is the same as

$$
\sum_{p}\left|\frac{p^{-(s+1)}}{\left(1-p^{-(s+1)}\right)}\right|=\sum_{p}\left|p^{-(s+1)}+\frac{p^{-2(s+1)}}{\left(1-p^{-(s+1)}\right)}\right| .
$$

Hence $I_{f}(s)$ converges absolutely if and only if $\Re(s)>0$.

A first meromorphic continuation in general:

Proposition 7 Let $f(x, y) \in \mathbb{Z}[x, y]$ be a non-singular polynomial then $I_{f}(s)$ has a meromorphic continuation to the region $\Re(s)>-1 / 2$.

Proof. Firstly

$$
\left(1-p^{-(s+1)}\right)\left(1+\lambda_{p} \frac{p^{-(s+1)}}{\left(1-p^{-(s+1)}\right)}\right)=1-p^{-(s+1)}+\lambda_{p} p^{-(s+1)}
$$

and

$$
\begin{aligned}
-p^{-(s+1)}+\lambda_{p} p^{-(s+1)} & =\frac{(p-1)\left(p-a_{p}\right) p^{-(s+1)}-\left(p^{2}-p+a_{p}\right) p^{-(s+1)}}{\left(p^{2}-C_{p}\right)} \\
& =\frac{-a_{p} p^{-s}}{\left(p^{2}-C_{p}\right)} .
\end{aligned}
$$


Thus writing

$$
I_{E}(s)=\zeta(s+1) \prod_{p}\left(1-\frac{a_{p} p^{-s}}{\left(p^{2}-C_{p}\right)}\right)
$$

continues $I_{f}(s)$ up to $\Re(s)>-1 / 2$.

The results of this section up to this point are considered by Ono in his paper $[12]$.

From now on we specialize to the case when our non-singular polynomial $f(x, y) \in \mathbb{Z}[x, y]$ defines an elliptic curve $E$, that is $g(f)=1$. We first introduce some Dirichlet series related to the global Igusa zeta function $I_{f}(s)$. First of all we recall the definition of the Hasse-Weil zeta function of $E$. Let $\tilde{E}$ be the projectivized elliptic curve $E$ and $N_{p^{m}}$ the number of points in $\tilde{E}\left(\mathbb{F}_{p^{m}}\right)$. Put

$$
\zeta(E, p, s)=\exp \left(\sum_{m=1}^{\infty} N_{p^{m}} \frac{p^{-m s}}{m}\right)
$$

Note that this is the projective version of the curve in which case $N_{p}=C_{p}+1$ since there is one extra point at infinity not picked up by $C_{p}$. We then have the following explicit formula for this zeta function:

$$
\zeta(E, p, s)=\frac{1-a_{p} p^{-s}+p^{1-2 s}}{\left(1-p^{-s}\right)\left(1-p^{1-s}\right)} .
$$

The Hasse-Weil zeta function is defined by

$$
\zeta(E, s)=\prod_{p \notin S(f)}\left(1-a_{p} p^{-s}+p^{1-2 s}\right)^{-1} .
$$

It is known that the Euler product of these zeta functions is meromorphic. We recall some facts about $a_{p}$ :

\section{Proposition $8 \quad$ (i) $\left|a_{p}\right| \leq 2 p^{1 / 2}$}

(ii) There exist algebraic integers $\pi_{p}$ and $\bar{\pi}_{p}$ in some finite extension of $\mathbb{Q}$ with $\pi_{p} \overline{\pi_{p}}=p$ and $a_{p}=\pi_{p}+\overline{\pi_{p}}$ and $\left|\pi_{p}\right|=\left|\overline{\pi_{p}}\right|=p^{1 / 2}$.

Suppose now that $E$ has complex multiplication. This means that its ring of endomorphisms $\operatorname{End}_{\mathbb{C}}(E)$ is not equal to $\mathbb{Z}$ only. Consequently the tensor product $K:=\mathbb{Q} \otimes_{\mathbb{Z}} \operatorname{End}_{\mathbb{C}}(E)$ is an imaginary quadratic number field. Furthermore there is a choice of the $\pi_{p}$ and an idele class character

$$
\chi_{E}: K^{*} \backslash \mathbb{A}_{k}^{*} \rightarrow \mathbb{C}^{*}
$$

such that

$$
\zeta(E, p, s)=1-a_{p} p^{-s}+p^{1-2 s}=\prod_{\wp \mid p}\left(1-\chi([\wp]) N(\wp)^{-s}\right)
$$

If $E$ does not have complex multiplication then by Wiles theorem there is a holomorphic modular form $g$ of weight 2 such that $\zeta(E, s)=L(s, g, 1)$.

We want to show next that we can continue $I_{E}(s)$ meromorphically to $\Re(s)>$ $-3 / 2$. We shall use the following functions to do the continuation which we have proved are meromorphic in section 3 . 
Proposition 9 Let

$$
Z_{r}(s)=\prod_{p \notin S(f)} Z_{n, p}(s)
$$

where

$$
Z_{r, p}(s)=1-\left(\pi_{p}^{r}+{\overline{\pi_{p}}}^{r}\right) p^{-s}+p^{r-2 s}
$$

Then $Z_{r}(s)$ is a meromorphic function on $\mathbb{C}$.

In fact as we shall see it is enough actually to have that they are meromorphic on $\Re(s)>1 / 2$.

Using this proposition we can now prove:

Theorem 5 The global Igusa zeta function of an elliptic curve $I_{E}(s)$ has meromorphic continuation to $\Re(s)>-3 / 2$.

Proof. By Proposition 7 we need to show how to meromorphically continue:

$$
\prod_{p \notin S(f)}\left(1-\frac{a_{p} p^{-s}}{\left(p^{2}-C_{p}\right)}\right)
$$

We write for $p \notin S(f)$

$$
\left(1-\frac{a_{p} p^{-s}}{\left(p^{2}-C_{p}\right)}\right)=\left(1-a_{p} p^{-2-s}-\frac{a_{p} p^{-2-s}\left(p^{-1}-a_{p} p^{-2}\right)}{\left(1-p^{-1}+a_{p} p^{-2}\right)}\right) .
$$

Since

$$
\sum_{p} \frac{a_{p} p^{-2-s}\left(p^{-1}-a_{p} p^{-2}\right)}{\left(1-p^{-1}+a_{p} p^{-2}\right)}
$$

converges absolutely on $\Re(s)>-3 / 2$ it will suffice to prove that

$$
W(s)=\prod_{p \notin S(f)}\left(1-a_{p} p^{-s}\right)
$$

can be meromorphically continued to $\Re(s)>1 / 2$.

By Lemma 1 we can write for $M \in \mathbb{N}$ :

$$
\begin{aligned}
\prod_{p \notin S(f)}\left(1-a_{p} p^{-s}\right) & =\prod_{p \notin S(f)}\left(1-\left(\pi_{p}+\overline{\pi_{p}}\right) p^{-s}\right) \\
& =\prod_{p \notin S(f)}\left(Q_{M}\left(\pi_{p}, \overline{\pi_{p}}, p, p^{-s}\right)+W_{M}\left(\pi_{p}, \overline{\pi_{p}}, p, p^{-s}\right)\right) \\
& =\left(\prod_{p \notin S(f)} Q_{M}\left(\pi_{p}, \overline{\pi_{p}}, p, p^{-s}\right)\right) \prod_{p \notin S(f)}\left(1+\frac{W_{M}\left(\pi_{p}, \overline{\pi_{p}}, p, p^{-s}\right)}{Q_{M}\left(\pi_{p}, \overline{\pi_{p}}, p, p^{-s}\right)}\right)
\end{aligned}
$$

Recall $Q_{M}$ was defined as a product of the polynomials

$$
P_{(r, n, m)}^{[\varepsilon]}=1-\varepsilon\left(u^{r}+v^{r}\right) X^{n} Y^{m}+X^{r+2 n} Y^{2 m}
$$

Now

$$
P_{(r, n, m)}^{[\varepsilon]}\left(\pi_{p}, \overline{\pi_{p}}, p, p^{-s}\right)=1-\left(\pi_{p}^{r}+{\overline{\pi_{p}}}^{r}\right) p^{-m s+n}+p^{r-2 s}=Z_{r, p}(m s-n)
$$


By Proposition 9 this implies that

$$
\left(\prod_{p \notin S(f)} Q_{M}\left(\pi_{p}, \overline{\pi_{p}}, p, p^{-s}\right)\right)
$$

is a meromorphic function on $\mathbb{C}$.

By Lemma 2

$$
\prod_{p \notin S(f)}\left(1+\frac{W_{M}\left(\pi_{p}, \overline{\pi_{p}}, p, p^{-s}\right)}{Q_{M}\left(\pi_{p}, \overline{\pi_{p}}, p, p^{-s}\right)}\right)
$$

converges absolutely for $\Re(s)>1 / 2+1 / M$.

Therefore by taking $M$ to infinity we can meromorphically continue $W(s)$ to $\Re(s)>1 / 2$. This in turn implies that the global Igusa zeta function of an elliptic curve $I_{E}(s)$ has meromorphic continuation to $\Re(s)>-3 / 2$.

\section{$5 \quad$ Natural boundaries}

In this section we let $f(x, y) \in \mathbb{Z}[x, y]$ be a nonsingular integer polynomial defining an elliptic curve $E$. Recall that we did our meromorphic continuation using the function

$$
\prod_{p \notin S(f)} Q_{M}\left(\pi_{p}, \overline{\pi_{p}}, p, p^{-s}\right) .
$$

This was built from a product and quotient of symmetric power L-functions which by [1, 9] and [14] are meromorphic on $\mathbb{C}$. Therefore the possible poles of our meromorphic function doing the continuation depends on the location of the poles and zeros of the symmetric power L-functions. Working under the assumption of the Generalized Riemann Hypothesis we can assume that zeros are located on $\Re(s)=1 / 2$. It will be sufficient to know that any poles of the symmetric power L-functions lie on the real axis.

Interest in meromorphically continuing the symmetric power $L$-functions arose because of Serre's observation that this would imply the Sato-Tate conjecture for the distribution of the values of $a_{p}$ in the case of $E$ not having complex multiplication. The distribution in the case of CM curves was known from class field theory.

In particular the results of [1], 9] and [14] imply the truth of the following:

Theorem 6 Suppose $E$ has no complex multiplication. Then $a_{p} p^{-1 / 2} / 2$ is equidistributed in $[-1,1]$ with respect to the probability measure $\frac{2}{\pi} \sqrt{1-t^{2}}$.

In all cases we have the following:

Theorem 7 For each elliptic curve $E$ there is an infinite set $\mathcal{P}_{E}$ of primes $p$ such that $a_{p} / p^{-1 / 2}>1$.

Theorem 8 Assume that $I_{f}(s)$ has a meromorphic continuation to the whole of the region defined by $\Re(s)>-3 / 2$ and also assume the generalized Riemann Hypothesis, then the line $\Re(s)=-3 / 2$ is a natural boundary for $I_{E}(s)$ beyond which no further meromorphic continuation is possible. 
Proof. We show that for infinitely many $p$ the equation

$$
1-\frac{a_{p} p^{-s}}{\left(p^{2}-C_{p}\right)}=0
$$

has a solutions at $s_{p}+\left(\theta_{p}+2 \pi n\right) i / \log p$ where $s_{p} \in \mathbb{R}, s_{p}>-3 / 2$. We also show that $s_{p} \rightarrow-3 / 2$ as $p \rightarrow \infty$. We then need to establish that for sufficiently large $p$ these zeros cannot be zeros or poles of the meromorphic functions being used to do the continuation.

Note first that for almost all primes $p$ we have $a_{p} \neq 0$ and (28) is satisfied if and only if

$$
p^{s}=p^{-\frac{3}{2}}\left(\frac{b_{p}}{1-p^{-1}+b_{p} p^{-\frac{3}{2}}}\right)
$$

where $b_{p}:=a_{p} p^{-\frac{1}{2}}$. Put

$$
r_{p}:=\frac{b_{p}}{1-p^{-1}+b_{p} p^{-\frac{3}{2}}} .
$$

By Theorem 7 there is an infinite set $\mathcal{P}_{E}$ of primes $p$ such that $b_{p}>1$ which in turn implies

$$
b_{p}>\frac{1-p^{-1}}{1-p^{-\frac{3}{2}}}
$$

for every $p \in \mathcal{P}_{E}$. Inequality (31) implies $r_{p}>1$, hence we have zeros of the form $s_{p}+\left(\theta_{p}+2 \pi n\right) i / \log p$ where $s_{p} \in \mathbb{R}, s_{p}>-3 / 2$. Since $r_{p}$ remains bounded as $p$ ranges over $\mathcal{P}_{E}$ we also have $s_{p} \rightarrow-3 / 2$ as $p \rightarrow \infty$ in $\mathcal{P}_{\epsilon}$.

We can therefore realise every point $-3 / 2+a i$ on the candidate natural boundary as a limit point of these local zeros by letting $p \rightarrow \infty$ and choosing $n_{p}$ with the property that $\left(\theta_{p}+2 \pi n_{p}\right) i / \log p \rightarrow a$.

Recall that the following expression was how we continued $I_{f}(s)$ to $\Re(s)>$ $-3 / 2$ by considering $M$ tending to infinity:

$$
I_{f}(s)=\prod_{p \notin S(f)} Q_{M}\left(\pi_{p}, \overline{\pi_{p}}, p, p^{-s}\right) \cdot \prod_{p \notin S(f)}\left(1+\frac{W_{M}\left(\pi_{p}, \overline{\pi_{p}}, p, p^{-s}\right)}{Q_{M}\left(\pi_{p}, \overline{\pi_{p}}, p, p^{-s}\right)}\right) .
$$

We therefore need to confirm that the limit points of zeros we have identified cannot be killed by poles or zeros of $Z_{r}(m s+n)$ or $\zeta(m s-n)$ where $n / m=-3 / 2$. We established that in fact the zeta function $Z_{n}(m s+n)$ can be rewritten in terms of the symmetric power $L$-functions so we only have to worry about zeros possibly cancelling our local zeros. Since we are assuming the generalized Riemann Hypothesis we can assume that a non-trivial zero $\rho$ of the Riemann zeta function or the symmetric power $L$-functions is of the form $1 / 2+\gamma i$. Suppose that $m s^{\prime}+n=\rho$ and $p^{-s^{\prime}}=-a_{p}^{-1} p^{2}+a_{p}^{-1} p-1$. Note that $-a_{p}^{-1} p^{2}+a_{p}^{-1} p-1$ is a rational number, whilst $p^{-s^{\prime}}=p^{-(1 / 2 m-3 / 2+\gamma i / m)}$. So the only possible value of $m$ for which this could happen is $m=1$. The only global zeros that can possibly interfere with local zeros are all on $\Re(s)=-1$.

This confirms then that $\Re(s)=-3 / 2$ is a natural boundary for $I_{E}(s)$. 


\section{References}

[1] L. Clozel, M. Harris, R. Taylor, Automorphy for some -adic lifts of automorphic mod Galois representations, Pub. Math. IHES 108, Issue 1 , (2008) pp 1-181.

[2] M.P.F. du Sautoy and Luke Woodward, Zeta Functions of Groups and Rings. Lecture Notes in Mathematics 1925 Springer (2008).

[3] M.P.F. du Sautoy and F.J. Grunewald, Analytic properties of zeta functions and subgroup growth Ann of Math. 152 (2000) 793-833.

[4] M.P.F. du Sautoy, A nilpotent group and its elliptic curve: non-uniformity of local zeta functions of groups. Isr. J. Math. 126 (2001) 269-288.

[5] M.P.F. du Sautoy, Counting subgroups in nilpotent groups and points on elliptic curves. J. REine. Angew. Math. 549 (2002) 1-21.

[6] T. Estermann, On certain functions represented by Dirichlet series, Proc. London Math. Soc. 27 (1928), 435-448.

[7] S. Gelbart, Automorphic forms on Adele groups, Ann. of Math. Studies, 83, Princeton University Press, Princeton (1975)

[8] F.J. Grunewald, D. Segal and G.C. Smith, Subgroups of finite index in nilpotent groups, Invent. Math., 93 (1988), 185-223.

[9] M. Harris, N. Shepherd-Barron, R. Taylor, A family of Calabi-Yau varieties and potential automorphy, Ann. of Math, 171 (2010) 779-813.

[10] S. Lang, Algebraic number theory, Graduate Text in Math., 110, SpringerVerlag, Berlin-Heidelberg-New York, (1970), Second Edition

[11] R. Langlands, Problems in the theory of automorphic forms, Lecture Notes in Math., 170, Springer-Verlag, Berlin-Heidelberg-New York, (1970), 18-86

[12] T. Ono, An integral attached to a hypersurface, Amer. J. Math., 90, (1968), 1224-1236.

[13] F. Shahidi, Symmetric Power L-functions for GL(2), in Elliptic curves and related topics, ed. by H. Kisilevsky, M. Ram Murty, CRM Proceedings and Lecture Notes, 4, 159-182, Amer. Math. Soc. (1994)

[14] R. Taylor, Automorphy for some -adic lifts of automorphic mod Galois representations II, Pub. Math. IHES 108 (2008), 183-239. 